\title{
Traduire
}

Une autre perspective sur r tr traduction

Revue française de la traduction

$244 \mid 2021$

Des jeux et des mots

\section{Les jeux vidéo en VF. La perspective d'un traducteur-joueur}

\section{Raphaël Rouby}

\section{(2) OpenEdition}

1 Journals

Édition électronique

URL : https://journals.openedition.org/traduire/2304

DOI : 10.4000/traduire.2304

ISSN : 2272-9992

Éditeur

Société française des traducteurs

Édition imprimée

Date de publication : 15 juin 2021

Pagination : 41-50

ISSN : 0395-773X

\section{Référence électronique}

Raphaël Rouby, «Les jeux vidéo en VF. La perspective d'un traducteur-joueur », Traduire [En ligne],

244 | 2021, mis en ligne le 15 juin 2021, consulté le 01 juillet 2021. URL : http://

journals.openedition.org/traduire/2304; DOI : https://doi.org/10.4000/traduire.2304 


\section{Les jeux vidéo en VF}

La perspective d'un traducteur-joueur

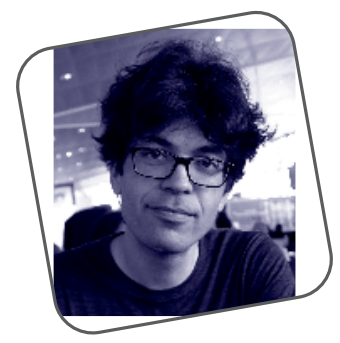

Raphaël Rouby

Contrairement à de nombreux fans qui disent préférer les jeux vidéo en version originale, selon eux plus "authentiques», je joue presque exclusivement en français. J'aime voir le résultat du travail de localisation.

Dans ces quelques pages, je vais utiliser mon expérience des deux côtés de l'interface pour proposer un regard de linguiste sur ce divertissement. Au cours de cette analyse très informelle, je donnerai des exemples tirés de ma pratique. Voici quelques questions abordées dans cet article: est-il indispensable d'être un as du joystick pour traduire? Que peut apporter la pratique vidéoludique à la traduction? Le traducteur est-il un gamer comme les autres? Parvient-il à mettre de côté son dictionnaire et son Bescherelle lorsqu'il enfile son armure ou obtient des superpouvoirs? Retire-t-il un plaisir supplémentaire si la localisation est de qualité?

Le fil directeur de l'article sera, et c'est assumé, mon expérience subjective. 


\section{Pour traduire des jeux vidéo, faut-il soi-même jouer?}

La réponse semble être dans la question... Pourtant, sans avoir fait d'étude sociologique, je pense pouvoir affirmer que ce n'est pas forcément le cas. En effet, je connais de nombreux collègues qui traduisent très bien et travaillent sur de beaux projets, sans pour autant passer leurs semaines manette à la main, voire en ne jouant jamais. La localisation de jeux vidéo est un travail de création d'univers: elle demande avant tout de l'inventivité, de l'adaptabilité stylistique et une grande maîtrise linguistique. Les compétences requises sont d'abord littéraires; I'habileté à la manette ne confère pas de légitimité professionnelle a priori.

Après cet indispensable rappel, voici donc ma propre expérience: je joue depuis l'âge de 11 ans (d'abord occasionnellement, puis plus régulièrement depuis mes études de traduction). Certains jeux ont façonné ma personnalité, au même titre que certains livres. Aujourd'hui, en tant que traducteur, je trouve naturel et utile de jover, car rien ne remplace le contact direct avec le média. Sans cette expérience, je me sentirais moins légitime. Et puis, reconnaissonsle: jover me plaît, tout simplement. Plonger dans un univers vidéoludique, c'est comme ouvrir un livre, en plus interactif... Et j'aime qu'on me raconte de belles histoires.

Un traducteur retire plusieurs avantages techniques et professionnels de ses sessions de jeu. Découvrir des univers manette à la main sensibilise aux contraintes de la localisation. Par exemple, on fait l'expérience visuelle de la taille limitée des éléments d'interface (menus, boutons), on picore parfois des idées de traduction pour des noms de bâtiments, de lieux ou de monstres, voire pour des abréviations. Autre avantage: on s'imprègne profondément des univers. Par exemple, Banner Saga permet une approche originale du monde viking, que ce soit par l'esthétique ou la mythologie. Enfin, en pratiquant, on découvre en contexte la terminologie du domaine (arbre de compétences dans les jeux de rôle, arbre des technologies dans les titres $4 X^{1}$, lobbys dans les

1. Jeux de gestion d'empire utilisant les mécaniques d'eXploration, d'eXpansion, eXploitation et extermination. 
jeux multijoueur). On fait l'expérience du gameplay in vivo: ainsi, on ne comprendra vraiment les ressorts de Mario Kart qu'en y jouant. Autre exemple des bénéfices liés à la pratique vidéoludique: je comprends que "taux d'attaque/taux de défense» puisse choquer les utilisateurs, plus habitués à un simple "attaque/défense» pour les statistiques ou les descriptions de compétences. Ou je sais d'expérience qu'il faut créer des sous-titres simples et concis lors des phases actives de jeu, afin d'éviter la surcharge cognitive de l'utilisateur, déjà concentré sur la coordination entre les commandes qu'il donne et ce qu'il voit à l'écran.

\section{Le traducteur, un joueur comme un autre?}

Là encore, la question est volontairement provocante.

Lorsque j'ai participé à la localisation de Warhammer Online en français, j'ai passé de nombreux soirs à jouer pour me familiariser avec ce MMORPG ${ }^{2}$. Au bout de quelques heures, je passais rapidement sur les textes des innombrables quêtes: je lisais la version résumée, ou pire, simplement la liste des objets à obtenir, pour aller un peu plus vite. J'étais devenu comme n'importe quel joueur qui veut faire progresser son personnage sans se perdre dans un processus déjà ultra-chronophage. Pourtant, ma mauvaise conscience me taraudait: en tant que traducteur, je savais le temps et le soin requis pour la traduction de ces quêtes; de plus, c'était un jeu sur lequel je travaillais!

Mon intérêt pour le côté linguistique des jeux ne s'arrête pas aux textes eux-mêmes. En effet, je suis devenu sensible au dosage entre le gameplay ${ }^{3}$ et les textes. Quand ce mélange est réussi, il passe inapercu, à l'instar d'une bonne traduction. Par exemple, dans Octopath Traveler, I'histoire est très présente en filigrane, sans être jamais envahissante. Les concepteurs doivent trouver un savant équilibre entre

2. Acronyme signifiant «jeu de rôle massivement multijoueur» (World of Warcraft étant le plus célèbre). Pour plus de précisions, voir glossaire général.

3. Ou «jouabilité», en français. II s'agit de l'ensemble des mécaniques de fonctionnement d'un jeu. Pour plus de précisions, voir glossaire général. 
le grinding ${ }^{4}$ bête et méchant non contextualisé, assez frustrant, et un enrobage scénaristique trop foisonnant. Parfois, les jeux ultra-scénarisés se rapprochent des livres dont vous êtes le héros ou des fictions interactives des années 1970. En début de carrière, j'ai relu un manuel de la franchise Final Fantasy. J'avais lancé le jeu pour vérifier certaines commandes, et la cinématique d'introduction m'avait paru interminable, car j'avais peu de temps dans ce contexte professionnel. En tant que traducteur, je trouve qu'un excellent gameplay sans texte manque parfois de relief (même si ce parti pris d'économie textuelle peut être très réussi, comme dans Machinarium). À l'inverse, un jeu très bavard aux rares interactions (Chrono Trigger) se mue en expérience narrative, avec le risque de perdre en interactivité et en plaisir ludique.

Bien sûr, un traducteur n'est pas seulement sensible à la place de la narration dans un jeu; il remarque aussi les textes eux-mêmes.

\section{Splendeurs et misères de la localisation}

Je vais vous donner quelques exemples issus de mon expérience de joveur. Afin d'éviter un inventaire à la Prévert, j'organiserai cette section en trois thèmes: des jeux dont la traduction m'a impressionné, des exemples d'erreurs de localisation et, enfin, un retour sur de célèbres erreurs de localisation.

Le Bon: quand je joue, je suis souvent admiratif des trouvailles des confrères et consœurs, et je savoure les belles traductions; je ne passe pas mon temps à me demander comment j'aurais fait à leur place ni à chercher une meilleure tournure. Ainsi, cette phrase d'Octopath Traveller m'a suffisamment plu pour que j'en fasse une capture d'écran et que je songe à l'intégrer à un article de blog.

4. Répétition pratiquement à l'infini d'une action permettant de faire progresser l'expérience de son personnage ou d'augmenter ses ressources: par exemple, tuer un monstre. 


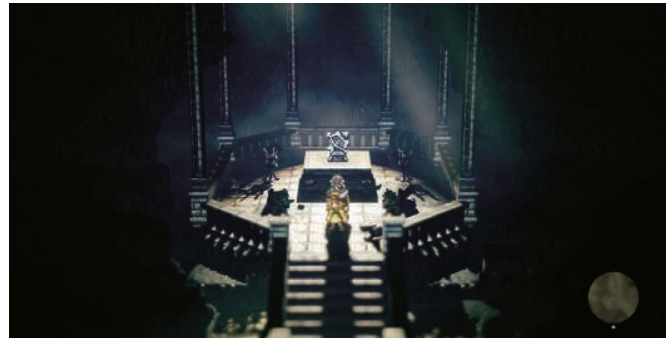

"À vous qui avez bravé le danger pour arriver jusqu'ici, je transmets le savoir des cieux." Une formulation élégante peut me rendre aussi heureux que d'avoir triomphé d'un boss! (c) Square Enix/Acquire

Aujourd'hui encore, plus de dix ans après avoir joué à Dragon Age: Origins, je me souviens de Grand Chêne, ce sylvan de la forêt de Bréciliane qui parlait en alexandrins.

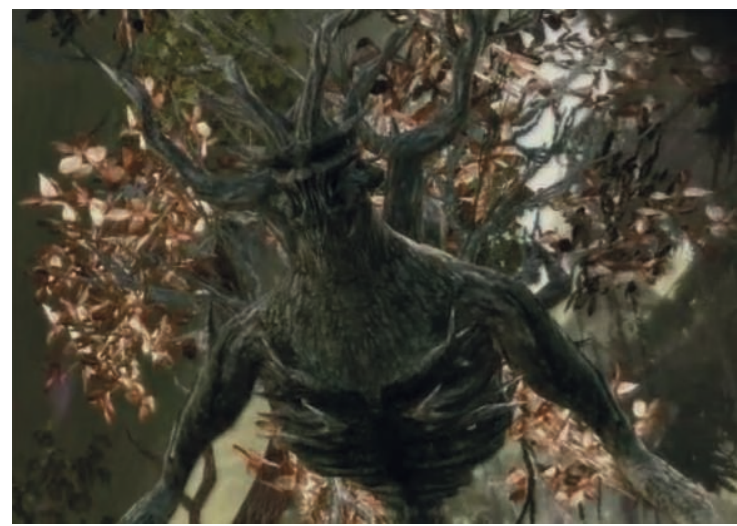

«Mon fruit, mon précieux fruit me fait toujours défaut, Pourquoi donc, mon ami, revenez-vous si tôt? Ô joie, ô grand bonheur, soyez porté aux nues! Grâce à votre concours, mon fruit m'est revenu." (C) Bioware/EA

Le dialogue avec ce $\mathrm{PNJ}^{5}$ se poursuit pendant plusieurs minutes: le travail d'adaptation a dû être colossal. Ayons une petite pensée émue et admirative pour la personne à l'origine de ces vers. Je les imagine traduits en quelques journées (ou nuits?) stressantes... car lorsque l'on calcule les plannings en tablant sur 2000 ou 2500 mots par jour, le rythme de travail

5. Personnage non joueur: désigne tous les personnages que le joueur n'incarne pas, mais qui peuplent les univers et avec lesquels il interagit (donneurs de quête, marchands, ennemis...). 
demandé est peu propice à la poésie. J'aurais aimé pouvoir te rendre hommage nommément, ô collègue; hélas, la localisation du jeu a été confiée à une agence et je ne connaîtrai jamais ton nom. (II est regrettable que les clauses de confidentialité empêchent souvent d'intégrer dans le générique les noms des autrices ou auteurs des traductions. II serait normal de nommer systématiquement tous les membres de l'équipe de localisation, comme il est d'usage pour un soustitrage ou une traduction d'édition. Déjà qu'un traducteur de jeux vidéo ne touche pas de droits d'auteur...)

Troisième exemple, toujours dans cette veine poétique: Child of Light, entièrement rimé! La traduction de Céline Le Roy est fluide, pleine de finesse et de justesse. Cette narration originale a d'ailleurs inspiré les commentaires des joveurs sur la plateforme de téléchargement Steam, ou ce journaliste qui a écrit son test intégralement en vers...

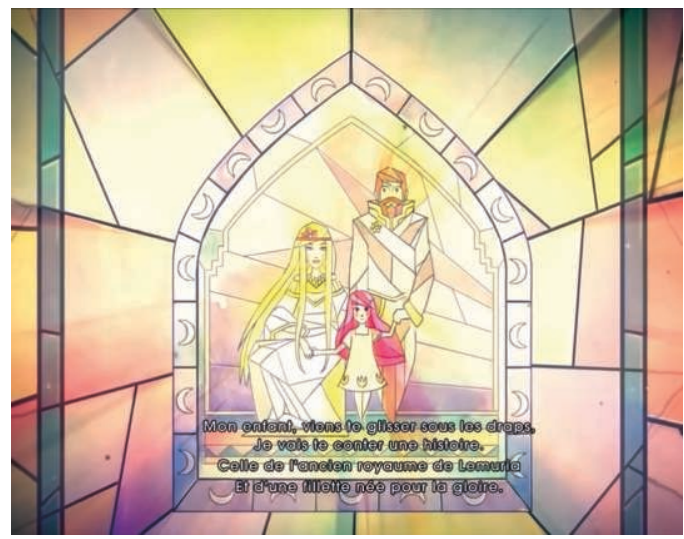

(C) Ubisoft

La Brute: la plupart du temps, j'ai donc plaisir à jover, sauf quand les problèmes de traduction sont omniprésents. Entendons-nous bien: je ne parle pas seulement du style, mais d'erreurs de traduction si gênantes qu'elles nuisent à l'immersion et poussent à quitter rapidement le jeu... II ne s'agit pas de jeter la pierre; je ne suis pas non plus infaillible, et il faut remettre ces erreurs en perspective: manque de temps, budget trop restreint qui empêche de recruter des professionnels qualifiés (ou qui prive des personnes par ailleurs qualifiées du temps nécessaire à une bonne traduction), 
absence de relecture ou de test linguistique, traductions demandées sans fournir aucun contexte, dans le désordre, absence d'instructions claires ou de réponses aux questions des traducteurs... Quand les astres ne sont pas alignés, le résultat peut être catastrophique.

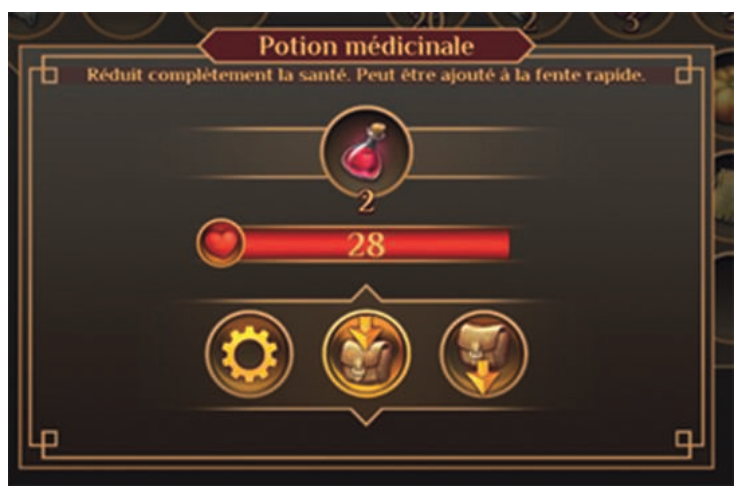

Dans Quest Hunter, la potion médicinale s'accorde au masculin on la met dans une "fente» (slot?).

Cerise sur le gâteau, elle réduit la santé...

C'est ce que I'on appelle un remède de cheval!

(c) 2 Zombie Games

Autre exemple emblématique, tiré d'une série qui a marqué I'histoire du jeu de rôle: la cinématique d'introduction de Baldur's Gate Shadows of Amn et son fameux «il fuya». Ce texte a sans doute été relu par un tiers, puis jové par le comédien en studio (normalement encadré par une directrice ou un directeur artistique).

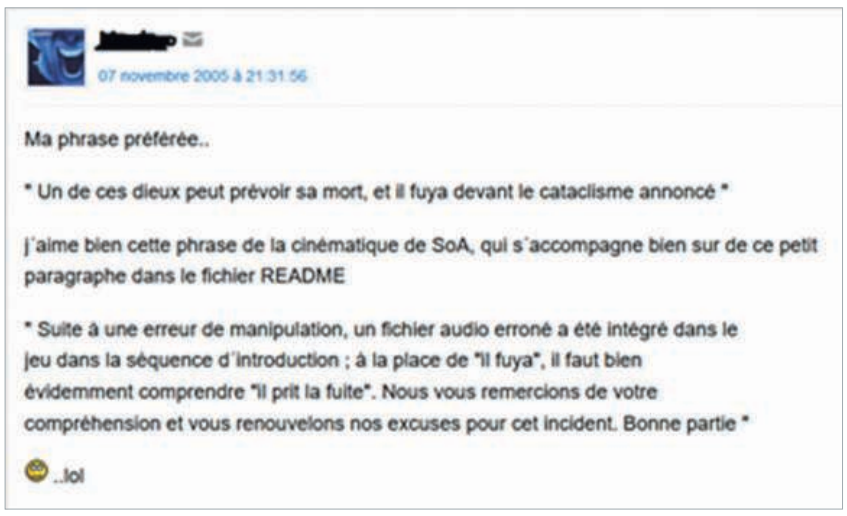


Précisons que cette erreur n'est absolument pas représentative de la qualité globale de la localisation, que j'avais trouvée excellente. La faute est bien excusable si l'on prend en compte le volume traduit, mais il est remarquable qu'elle ait traversé toutes les mailles du filet, jusqu'à être interprétée en studio, puis conservée à l'issue du test linguistique, alors que sa visibilité en jeu est maximale: elle se trouve dans la cinématique d'introduction. Sans doute était-il trop tard pour refaire l'enregistrement à temps pour la sortie. J'étais alors étudiant en traduction et je me souviens que cela m'avait fait bondir sur ma chaise: un traducteur n'est décidément pas un joueur comme les autres...

Le Truand: je me devais de trouver une correspondance, même ténue, avec le troisième larron de Sergio Leone. Dans ces lignes, je vais me faire l'avocat du diable et tenter d'aborder les problèmes de traduction sous un angle différent. Au cours des recherches effectuées pour cet article, j'ai été peiné de constater que parfois, la communauté n'hésitait pas à se moquer d'une coquille (certes répétée) perdue au milieu des centaines de milliers de mots que compte Elder Scrolls IV: Oblivion.

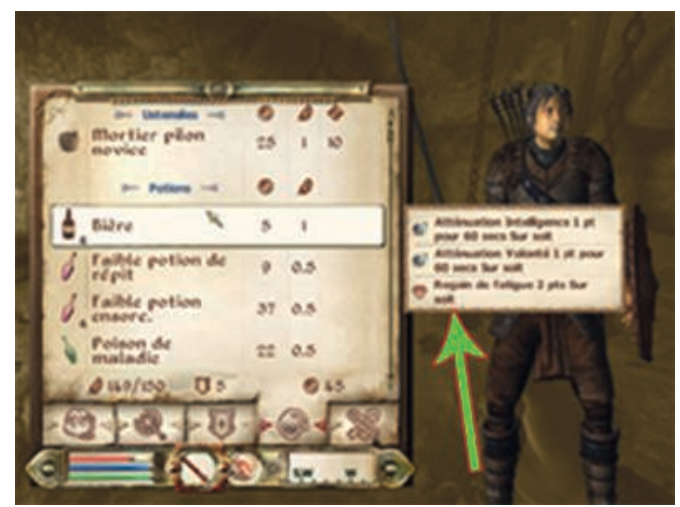

«Regain de fatigue 2 pts sur soit»

Certes, la faute est vilaine, mais il ne faut pas oublier que le volume traduit est considérable (exemple tiré d'un bêtisier paru sur le site Grospixels).

Les joueurs ne sont pas toujours conscients des contraintes de la localisation ni de la pression subie par le traducteur au cours d'un projet. Bien sûr, il n'est pas question de s'enfermer dans une tour d'ivoire, mais cette mise en perspective s'impose. 
Terminons cet inventaire par une localisation ratée si retentissante qu'elle a donné naissance au mème "All your base are belong to us». II s'agit de Zero Wing, mal adapté depuis le japonais vers l'anglais. L'introduction du jeu a peut-être été écrite par les développeurs japonais eux-mêmes, qui n'ont pas su (ou pu?) faire appel à des traducteurs professionnels, de langue maternelle anglaise. Et cette phrase sans queve ni tête est passée à la postérité.

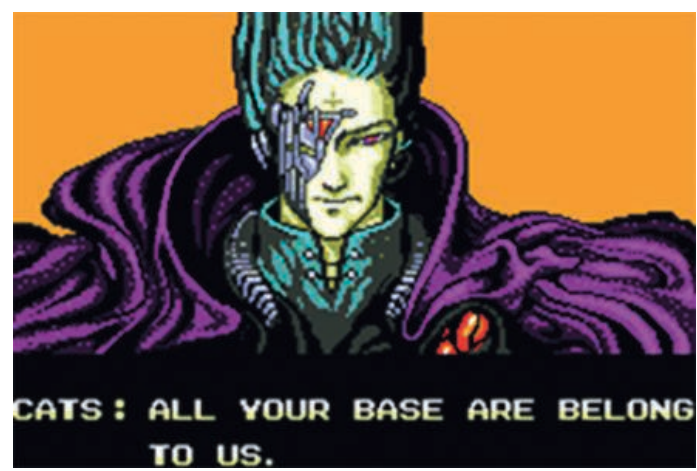

Le célèbre mème a été réintroduit dans de nombreux jeux vidéo, sous forme de référence.

Nous venons de le voir: les linguistes que nous sommes ont bien du mal à garder un œil strictement neutre en jouant. Cependant, quand la magie opère, nous oublions le côté technico-linguistique pour nous laisser happer par de belles histoires.

Je terminerai par un clin d'œil à l'ensemble de la profession. Il est tiré de Civilization VI, le dernier opus d'une série que j'apprécie depuis ses débuts. Les développeurs ont eu l'idée de faire parler les leaders politiques des différentes factions en version originale sous-titrée. Même si les personnages parlent parfois avec l'accent anglais (Aliénor d'Aquitaine ou Catherine de Médicis...), l'immersion historique est au rendez-vous. Trajan s'exprime en latin, Périclès en grec ancien, Pierre le Grand en russe... J'y vois un hommage à la diversité culturelle et historique de l'humanité, et cela me renvoie à notre travail de passeurs de mots. 
Titulaire d'une maîtrise d'histoire contemporaine et d'un master 2 de traduction à I'ITIRI, Raphaël Rouby est traducteur depuis 2003. II s'est spécialisé dans les jeux vidéo et la communication d'entreprise. II a travaillé en interne pour des éditeurs en France et en Irlande (Electronic Arts, Goa Games, Big Fish Games), et dans une agence de localisation en Angleterre. II travaille depuis plusieurs années en tant que traducteur freelance, traitant désormais principalement en direct avec les éditeurs. Quelques expériences en gestion de projet lui ont confirmé qu'il préférait jongler avec les mots qu'avec les tâches.

Gros Pixels, Le bêtisier des jeux vidéo, https://www.grospixels.com/site/betisier. php (site recensant des problèmes de traduction, principalement liés à l'absence de contexte), consulté le 28/04/2021.

BOUQUET Robin "Raiden Robin", Jeux vidéo: les ratés de la localisation, MMG (Millenium), 2 janvier 2019, https://fr.millenium.gg/news/133811.html (site consacré aux problèmes de localisation, donnant un aperçu de notre métier), consulté le $28 / 04 / 2021$.

Mini-ludographie sélective et subjective de jeux que j’ai trouvés bien localisés: Dragon Quest XI

Call of Juarez

Child of Light

Plants vs Zombies

Red Dead Redemption II 